\title{
NOTES ON THE DISTRIBUTION OF ROOTS MODULO A PRIME OF A POLYNOMIAL III
}

\author{
YOSHIYUKI KITAOKA \\ Author is retired, Asahi, JAPAN
}

\begin{abstract}
Let $f(x)$ be a monic polynomial with integer coefficients and integers $r_{1}, \ldots, r_{n}$ with $0 \leq r_{1} \leq \cdots \leq r_{n}<p$ the $n$ roots of $f(x) \equiv 0 \bmod p$ for a prime $p$. We proposed conjectures on the distribution of the point $\left(r_{1} / p, \ldots, r_{n} / p\right)$ in the previous papers. One aim of this paper is to revise them for a reducible polynomial $f(x)$, and the other is to show that they imply the one-dimensional equidistribution of $r_{1} / p, \ldots, r_{n} / p$ for an irreducible polynomial $f(x)$ by a geometric way.
\end{abstract}

\section{Communicated by Shigeki Akiyama}

Throughout this paper, a polynomial means a monic one over the $\operatorname{ring} \mathbb{Z}$ of integers and the letter $p$ denotes a prime number. Let

$$
f(x)=x^{n}+a_{n-1} x^{n-1}+\cdots+a_{0}
$$

be a polynomial of degree $n$ with complex roots $\alpha_{1}, \ldots, \alpha_{n}$. We fix the numbering of roots once and for all, and define a vector space $L R$ over the rational number field $\mathbb{Q}$ by

$$
L R:=\left\{\left(l_{1}, \ldots, l_{n+1}\right) \in \mathbb{Q}^{n+1} \mid \sum_{i=1}^{n} l_{i} \alpha_{i}=l_{n+1}\right\},
$$

which depends on the numbering of roots $\alpha_{i}$. The projection defined by

$$
\left(l_{1}, \ldots, l_{n}, l_{n+1}\right) \mapsto\left(l_{1}, \ldots, l_{n}\right)
$$

from $L R$ to

$$
L R_{0}:=\left\{\left(l_{1}, \ldots, l_{n}\right) \in \mathbb{Q}^{n} \mid \sum_{i=1}^{n} l_{i} \alpha_{i} \in \mathbb{Q}\right\}
$$

(C) 2020 BOKU-University of Natural Resources and Life Sciences and Mathematical Institute, Slovak Academy of Sciences.

2010 Mathematics Subject Classification: $11 \mathrm{~K}$.

Keywords: Equidistribution, polynomial, roots modulo a prime.

Licensed under the Creative Commons Attribution-NC-ND 4.0 International Public License. 


\section{YOSHIYUKI KITAOKA}

is an isomorphism, more strongly from $L R \cap \mathbb{Z}^{n+1}$ on $L R_{0} \cap \mathbb{Z}^{n}$, since numbers $\alpha_{i}$ are algebraic integers. Since there is a trivial linear relation $\sum \alpha_{i}=-a_{n-1}$, the non-zero vector $\left(1, \ldots, 1,-a_{n-1}\right)$ is always in $L R$, hence $t:=\operatorname{dim}_{\mathbb{Q}} L R=$ $\operatorname{dim}_{\mathbb{Q}} L R_{0} \geq 1$ is clear. The inequality $t \leq n$ is also clear and the condition $t=n$ holds if and only if $f(x)$ is a product of linear forms in $\mathbb{Q}[x]$. The vector $(1, \ldots, 1)$ is always in $L R_{0}$, and a polynomial $f(x)$ has a rational root if and only if a vector of the form $(0, \ldots, 0,1,0, \ldots, 0)$ is in $L R_{0}$, and $\alpha_{i}-\alpha_{j} \in \mathbb{Q}$ holds for distinct $i, j$ if and only if a vector of the form $(0, \ldots, 0,1,0, \ldots, 0,-1,0, \ldots, 0)$ is in $L R_{0}$.

We say that a polynomial $f(x)$ has a non-trivial linear relation among roots if $t>1$.

We take a $\mathbb{Z}$-basis

$$
\hat{\boldsymbol{m}}_{j}:=\left(m_{j, 1}, \ldots, m_{j, n}, m_{j}\right)(j=1, \ldots, t)
$$

of $L R \cap \mathbb{Z}^{n+1}$, and put

$$
\boldsymbol{m}_{j}:=\left(m_{j, 1}, \ldots, m_{j, n}\right)(j=1, \ldots, t) .
$$

By definition, the vector $\hat{\boldsymbol{m}}_{j}$ satisfies

$$
\sum_{i=1}^{n} m_{j, i} \alpha_{i}=m_{j}(j=1, \ldots, t)
$$

and the vectors $\boldsymbol{m}_{1}, \ldots, \boldsymbol{m}_{t}$ are a basis of $L R_{0} \cap \mathbb{Z}^{n}$. We fix the basis $\hat{\boldsymbol{m}}_{j}$ $(j=1, \ldots, t)$ together with roots $\alpha_{i}$ once and for all.

We introduce two permutation groups associated with them:

$$
\begin{aligned}
& \hat{\boldsymbol{G}}:=\left\{\nu \in S_{n} \mid\left\langle\nu\left(\hat{\boldsymbol{m}}_{1}\right), \ldots, \nu\left(\hat{\boldsymbol{m}}_{t}\right)\right\rangle_{\mathbb{Z}}=\left\langle\hat{\boldsymbol{m}}_{1}, \ldots, \hat{\boldsymbol{m}}_{t}\right\rangle_{\mathbb{Z}}\right\}, \\
& \boldsymbol{G}:=\left\{\nu \in S_{n} \mid\left\langle\nu\left(\boldsymbol{m}_{1}\right), \ldots, \nu\left(\boldsymbol{m}_{t}\right)\right\rangle_{\mathbb{Z}}=\left\langle\boldsymbol{m}_{1}, \ldots, \boldsymbol{m}_{t}\right\rangle_{\mathbb{Z}}\right\} .
\end{aligned}
$$

Here,

$$
\left\langle\boldsymbol{z}_{1}, \ldots, \boldsymbol{z}_{k}\right\rangle_{\mathbb{Z}}:=\left\{a_{1} \boldsymbol{z}_{1}+\cdots+a_{k} \boldsymbol{z}_{k} \mid a_{1}, \ldots, a_{k} \in \mathbb{Z}\right\}
$$

and we let, for $\boldsymbol{x}=\left(x_{1}, \ldots, x_{n}\right) \in \mathbb{R}^{n}, x \in \mathbb{R}$ and a permutation $\nu \in S_{n}$

Note that

$$
\nu(\boldsymbol{x}):=\left(x_{\nu^{-1}(1)}, \ldots, x_{\nu^{-1}(n)}\right), \nu((\boldsymbol{x}, x)):=(\nu(\boldsymbol{x}), x) .
$$

$$
\hat{\boldsymbol{G}}=\left\{\nu \in S_{n} \mid \nu(L R)=L R\right\}, \boldsymbol{G}=\left\{\nu \in S_{n} \mid \nu\left(L R_{0}\right)=L R_{0}\right\},
$$

hence, groups $\hat{\boldsymbol{G}}, \boldsymbol{G}$ are independent of the choice of bases $\hat{\boldsymbol{m}}_{i}$ and $\hat{\boldsymbol{G}}$ is a subgroup of $\boldsymbol{G}$. If $f(x)$ is irreducible, then they are identical. However, they are not necessarily equal for a reducible polynomial.

We may take the vector $\left(1, \ldots, 1,-a_{n-1}\right)$ as $\hat{\boldsymbol{m}}_{1}$, and if $f(x)$ has no non-trivial linear relation among roots, then it is a basis of $L R \cap \mathbb{Z}^{n+1}$, hence $\hat{\boldsymbol{G}}=\boldsymbol{G}=S_{n}$. 
Next, we put

$$
\operatorname{Spl}_{X}(f):=\{p \leq X \mid f(x) \text { is fully splitting modulo } p\}
$$

for a positive number $X$ and $\operatorname{Spl}(f):=\operatorname{Spl}_{\infty}(f)$.

We require the following conditions on the local roots $r_{1}, \ldots, r_{n}(\in \mathbb{Z})$ of $f(x) \equiv 0 \bmod p$ for a prime $p \in \operatorname{Spl}(f)$ :

$$
\begin{aligned}
& f(x) \equiv \prod_{i=1}^{n}\left(x-r_{i}\right) \bmod p, \\
& 0 \leq r_{1} \leq r_{2} \leq \cdots \leq r_{n}<p .
\end{aligned}
$$

The condition (11) is nothing but the definition of $p \in \operatorname{Spl}(f)$, and (2) determines local roots $r_{i}$ uniquely. From now on, local roots $r_{i}$ are supposed to satisfy two conditions (1) and (2). The global ordering (2) to local roots is a key.

We know that for a sufficiently large prime $p \in \operatorname{Spl}(f)$, there is at least one permutation $\sigma \in S_{n}$ dependent on $p$ such that

$$
\sum_{i=1}^{n} m_{j, i} r_{\sigma(i)} \equiv m_{j} \bmod p \quad\left(1 \leq{ }^{\forall} j \leq t\right) .
$$

To state conjectures, we introduce the following notation corresponding to the condition (3)

$$
\operatorname{Spl}_{X}(f, \sigma):=\left\{p \in \operatorname{Spl}_{X}(f) \mid(\underline{3})\right\},
$$

which is independent of the choice of the basis $\hat{\boldsymbol{m}}_{i}$, and we define the density by

$$
\operatorname{Pr}(f, \sigma):=\lim _{X \rightarrow \infty} \frac{\# \operatorname{Spl}_{X}(f, \sigma)}{\# \operatorname{Spl}_{X}(f)} .
$$

We suppose that the limit exists. The existence of the limit is supported by computer experiment.

Next, we introduce geometric objects

$$
\begin{aligned}
\hat{\mathfrak{D}}_{n} & :=\left\{\left(x_{1} \ldots, x_{n}\right) \in[0,1]^{n} \mid 0 \leq x_{1} \leq \ldots \leq x_{n} \leq 1, \sum_{i=1}^{n} x_{i} \in \mathbb{Z}\right\}, \\
\mathfrak{D}(f, \sigma) & :=\left\{\left(x_{1} \ldots, x_{n}\right) \in[0,1]^{n} \mid \begin{array}{l}
0 \leq x_{1} \leq \cdots \leq x_{n} \leq 1, \\
\sum_{i=1}^{n} m_{j, i} x_{\sigma(i)} \in \mathbb{Z}\left(1 \leq{ }^{\forall} \leq t\right)
\end{array}\right\} \\
& =\left\{\boldsymbol{x} \in \hat{\mathfrak{D}}_{n} \mid(\sigma(\boldsymbol{l}), \boldsymbol{x}) \in \mathbb{Z} \quad\left({ }^{\forall} \boldsymbol{l} \in L R_{0} \cap \mathbb{Z}^{n}\right)\right\} .
\end{aligned}
$$




\section{YOSHIYUKI KITAOKA}

The dimension of $\hat{\mathfrak{D}}_{n}$ is $n-1$ and that of $\mathfrak{D}(f, \sigma)$ is less than or equal to $n-t$. In the following, the volume $\operatorname{vol}(\mathfrak{D}(f, \sigma))$ means that as an $(n-t)$-dimensional set, that is for a set $S$ in $\boldsymbol{v}+\mathbb{R}\left[\boldsymbol{v}_{1}, \ldots, \boldsymbol{v}_{n-t}\right]\left(\subset \mathbb{R}^{n}\right)$ with orthonormal elements $\boldsymbol{v}_{1}, \ldots, \boldsymbol{v}_{n-t} \in \mathbb{R}^{n}$, we identify $\boldsymbol{v}+\sum y_{i} \boldsymbol{v}_{i} \in S$ with a point $\left(y_{1}, \ldots, y_{n-t}\right) \in \mathbb{R}^{n-t}$. So, $\operatorname{vol}(\mathfrak{D}(f, \sigma))=0$ holds if $\operatorname{dim} \mathfrak{D}(f, \sigma)<n-t$.

In case that $f(x)$ is a product of linear forms, which is equivalent to $t=n$, $\operatorname{dim} \mathfrak{D}(f, \sigma)=0$ holds, hence the discussion about $\mathfrak{D}(f, \sigma)$ is almost meaningless.

If there is no non-trivial linear relation among roots, then $\mathfrak{D}(f, \sigma)$ is equal to $\hat{\mathfrak{D}}_{n}$ for every permutation $\sigma$.

The first conjecture is

ConjeCture 1. For a permutation $\sigma$ with $\operatorname{Pr}(f, \sigma)>0$, the ratio

$$
c=\frac{\operatorname{vol}(\mathfrak{D}(f, \sigma))}{\operatorname{Pr}(f, \sigma)}
$$

is independent of $\sigma$. If $\boldsymbol{G}=\hat{\boldsymbol{G}}$ holds, then two conditions $\operatorname{Pr}(f, \sigma)>0$ and $\operatorname{vol}(\mathfrak{D}(f, \sigma))>0$ are equivalent.

If (i) $f(x)$ is not a product of linear forms, (ii) $\alpha_{i}-\alpha_{j} \notin \mathbb{Q}$ for any distinct $i, j$, that is, $f(x) \neq F(x) g(x) g(x+a)$ for any $F(x), g(x) \in \mathbb{Q}[x], a \in \mathbb{Q}$ and (iii) $\operatorname{vol}(\mathfrak{D}(f, \sigma))=c \operatorname{Pr}(f, \sigma)$ holds for every $\sigma \in S_{n}$, then

$$
c=\sqrt{\operatorname{det}\left(\left(\boldsymbol{m}_{i}, \boldsymbol{m}_{j}\right)\right)} / \# \hat{\boldsymbol{G}}
$$

is known (cf. K4]), although it is not necessary here.

There is an example of a reducible polynomial $f(x)$ such that $\operatorname{Pr}(f, \sigma)=0$ but $\operatorname{vol}(\mathfrak{D}(f, \sigma))>0$ for some permutation $\sigma$ as follows, and Expectation $1^{\prime \prime}$ in [K1], K3] should be revised as above, not to exclude a reducible polynomial.

EXAMPLe. Let us consider the polynomial $f(x)=\left(x^{2}-2\right)\left((x-1)^{2}-2\right)$ with $\operatorname{roots} \alpha_{1}=\sqrt{2}, \alpha_{2}=1+\sqrt{2}, \alpha_{3}=-\sqrt{2}, \alpha_{4}=1-\sqrt{2}$. Then $t=3$ and the matrix $\left(m_{j, i}\right)$ and $m_{i}$ are

$$
\left(\begin{array}{cccc}
1 & 0 & 0 & 1 \\
1 & -1 & 0 & 0 \\
1 & 0 & 1 & 0
\end{array}\right), \quad\left(\begin{array}{c}
1 \\
-1 \\
0
\end{array}\right)
$$

and

$$
\begin{array}{r}
\boldsymbol{G}=\{[1,2,3,4],[1,2,4,3],[2,1,3,4],[2,1,4,3], \\
[3,4,1,2],[3,4,2,1],[4,3,1,2],[4,3,2,1]\}
\end{array}
$$

and

$$
\hat{\boldsymbol{G}}=\{[1,2,3,4],[3,4,1,2]\},
$$

where a permutation $\sigma$ is identified with the vector $[\sigma(1), \ldots, \sigma(4)]$ of images. 
Then we see that

$$
\mathfrak{D}(f, \sigma)= \begin{cases}\{(x, x, 1-x, 1-x) \mid 0 \leq x \leq 1 / 2\} & \text { if } \sigma \in \boldsymbol{G}, \\ \{(1 / 2,1 / 2,1 / 2,1 / 2)\} & \text { otherwise. }\end{cases}
$$

Two conditions $\operatorname{dim} \mathfrak{D}(f, \sigma)=1(=n-t)$ and $\sigma \in G$ are equivalent. We find

since $p \in \operatorname{Spl}(f, \sigma)$ means

$$
\operatorname{Pr}(f, \sigma)= \begin{cases}1 & \text { if } \sigma \in \hat{\boldsymbol{G}} \\ 0 & \text { otherwise }\end{cases}
$$

$$
r_{\sigma(1)}+r_{\sigma(4)}=p+1, r_{\sigma(1)}-r_{\sigma(2)}=-1, r_{\sigma(1)}+r_{\sigma(3)}=p
$$

for a sufficiently large $p$, which implies $\sigma \in \hat{\boldsymbol{G}}$. This is an example such that $\operatorname{Pr}(f, \sigma)=0$ but $\operatorname{vol}(\mathfrak{D}(f, \sigma))>0$ for $\sigma \in \boldsymbol{G} \backslash \hat{\boldsymbol{G}}$ by $\# \boldsymbol{G}=8, \# \hat{\boldsymbol{G}}=2$, and this supports Conjecture 1 .

The revision with respect to Expectation $1^{\prime}$ in $\mathrm{K} 4$ for a reducible polynomial $f(x)$ is

ConjeCture 2. Suppose that $\operatorname{Pr}(f, \sigma)>0, \operatorname{vol}(\mathfrak{D}(f, \sigma))>0$ for a permutation $\sigma$; then for a set $D=\overline{D^{\circ}} \subset[0,1)^{n}$, we have

$$
\begin{aligned}
\operatorname{Pr}_{D}(f, \sigma): & =\lim _{X \rightarrow \infty} \frac{\#\left\{p \in \operatorname{Spl}_{X}(f, \sigma) \mid\left(r_{1} / p, \ldots, r_{n} / p\right) \in D\right\}}{\# \operatorname{Spl}_{X}(f, \sigma)} \\
& =\frac{\operatorname{vol}(D \cap \mathfrak{D}(f, \sigma))}{\operatorname{vol}(\mathfrak{D}(f, \sigma))} .
\end{aligned}
$$

The suppositions $\operatorname{Pr}(f, \sigma)>0, \operatorname{vol}(\mathfrak{D}(f, \sigma))>0$ are added.

Next, we discuss the one-dimensional equidistribution of $r_{i} / p(1 \leq i \leq n, p \in$ $\operatorname{Spl}(f))$ for local roots $r_{i}$, that is, whether for $0 \leq a<1$,

$$
\lim _{X \rightarrow \infty} \frac{\sum_{p \in \operatorname{Spl}_{X}(f)} \#\left\{i \mid r_{i} / p \leq a, 1 \leq i \leq n\right\}}{n \cdot \# \operatorname{Spl}_{X}(f)}=a
$$

is true or not. We note that if $f(x)$ has a rational integral root $r_{0}$, then $r=r_{0}$ or $r=p+r_{0}$ is a local root according to $r_{0} \geq 0$ or $r_{0}<0$ for a large prime $p \in \operatorname{Spl}(f)$, hence $r / p$ tends to 0 or 1 , that is the one-dimensional equidistribution is false. So, we exclude a polynomial with a rational integral root. We showed that Conjectures above imply the one-dimensional equidistribution of roots $r_{i} / p$ for a polynomial which has no non-trivial linear relation among roots by calculation (cf. K2]). Here we show for a more general polynomial, e.g., an irreducible polynomial of degree larger than 1, that Conjectures 1, 2 imply the one-dimensional equidistribution of roots $r_{i} / p$ not based on hard calculation. 
Putting

$$
D_{i, a}:=\left\{\left(x_{1}, \ldots, x_{n}\right) \in[0,1)^{n} \mid x_{i} \leq a\right\}
$$

it is easy to see

$$
\begin{aligned}
& \sum_{p \in \operatorname{Spl}_{X}(f)} \#\left\{i \mid r_{i} / p \leq a, 1 \leq i \leq n\right\} \\
= & \sum_{p \in \operatorname{Spl}_{X}(f)} \#\left\{i \mid\left(r_{1} / p, \ldots, r_{n} / p\right) \in D_{i, a}\right\} \\
= & \sum_{i=1}^{n} \#\left\{p \in \operatorname{Spl}_{X}(f) \mid\left(r_{1} / p, \ldots, r_{n} / p\right) \in D_{i, a}\right\} \\
= & \sum_{i=1}^{n} \sum_{\sigma \in S_{n}} \#\left\{p \in \operatorname{Spl}_{X}(f, \sigma) \mid\left(r_{1} / p, \ldots, r_{n} / p\right) \in D_{i, a}\right\} / \# \hat{\boldsymbol{G}}+O(1)
\end{aligned}
$$

since we know by Proposition 2 in [K3] that if a prime $p \in \operatorname{Spl}(f)$ is sufficiently large, then there are exactly $\# \hat{\boldsymbol{G}}$ permutations $\sigma$ satisfying $p \in \operatorname{Spl}(f, \sigma)$. Therefore we have

$$
\begin{aligned}
& \lim _{X \rightarrow \infty} \frac{\sum_{p \in \operatorname{Spl}_{X}(f)} \#\left\{i \mid r_{i} / p \leq a, 1 \leq i \leq n\right\}}{n \# \operatorname{Spl}_{X}(f)} \\
= & \lim _{X \rightarrow \infty} \sum_{i=1}^{n} \frac{\sum_{\sigma \in S_{n}} \#\left\{p \in \operatorname{Spl}_{X}(f, \sigma) \mid\left(r_{1} / p, \ldots, r_{n} / p\right) \in D_{i, a}\right\}}{n \cdot \# \hat{\boldsymbol{G}} \cdot \# \operatorname{Spl}_{X}(f)} \\
= & \lim _{X \rightarrow \infty} \sum_{i=1}^{n} \frac{\sum_{\sigma \in S_{n}^{\prime}} \#\left\{p \in \operatorname{Spl}_{X}(f, \sigma) \mid\left(r_{1} / p, \ldots, r_{n} / p\right) \in D_{i, a}\right\}}{\# \operatorname{Spl}_{X}(f, \sigma)} \\
& \times \frac{\# \operatorname{Spl}_{X}(f, \sigma)}{n \cdot \# \hat{\boldsymbol{G}} \cdot \# \operatorname{Spl}_{X}(f)}
\end{aligned}
$$

where $S_{n}^{\prime}$ is a subset of $S_{n}$ consisting of permutations satisfying $\operatorname{Pr}(f, \sigma)>0$

$$
\begin{aligned}
& =\frac{1}{n \# \hat{\boldsymbol{G}}} \sum_{i=1}^{n} \sum_{\sigma \in S_{n}^{\prime}} \operatorname{Pr}_{D_{i, a}}(f, \sigma) \operatorname{Pr}(f, \sigma) \\
& =\frac{1}{n c \# \hat{\boldsymbol{G}}} \sum_{i=1}^{n} \sum_{\sigma \in S_{n}^{\prime}} \operatorname{Pr}_{D_{i, a}}(f, \sigma) \operatorname{vol}(\mathfrak{D}(f, \sigma)) \quad \text { (by Conjecture 1) } \\
& =\frac{1}{n c \# \hat{\boldsymbol{G}}} \sum_{i=1}^{n} \sum_{\sigma \in S_{n}^{\prime}} \operatorname{vol}\left(D_{i, a} \cap \mathfrak{D}(f, \sigma)\right) \quad \text { (by Conjecture 2). }
\end{aligned}
$$


We note that the dimension of the set

$$
\begin{aligned}
& D_{i, 0} \cap \mathfrak{D}(f, \sigma) \\
& =\left\{\left(x_{1}, \ldots, x_{n}\right) \mid 0 \leq x_{1} \leq \cdots \leq x_{n}<1, x_{i}=0, \sum_{k=1}^{n} m_{j, k} x_{\sigma(k)} \in \mathbb{Z}\left({ }^{\forall} j\right)\right\}
\end{aligned}
$$

is less than $n-t$, since the condition $x_{i}=0$ is independent of conditions $\sum_{k=1}^{n} m_{j, k} x_{\sigma(k)} \in \mathbb{Z}\left({ }^{\forall} j\right)$ by the assumption that $f(x)$ has no rational root. Therefore the above is equal to 0 at $a=0$, and to 1 at $a=1$ by

$$
c=\frac{\sum_{\operatorname{Pr}(f, \sigma)>0} \operatorname{vol}(\mathfrak{D}(f, \sigma))}{\sum \operatorname{Pr}(f, \sigma)}=\sum_{\operatorname{Pr}(f, \sigma)>0} \operatorname{vol}(\mathfrak{D}(f, \sigma)) / \# \hat{\boldsymbol{G}},
$$

using Corollary 2 in $\mathrm{K} 3$. Hence, once we have shown that it is a linear form in $a$, it is equal to $a$ on $[0,1)$, that is the distribution of $r_{i} / p$ is uniform. The difference between the above sum restricted on $S_{n}^{\prime}$ and the full sum

$$
\sum_{i=1}^{n} \sum_{\sigma \in S_{n}} \operatorname{vol}\left(D_{i, a} \cap \mathfrak{D}(f, \sigma)\right)
$$

is

$$
\sum_{i=1}^{n} \sum_{\operatorname{Pr}(f, \sigma)=0, \operatorname{vol}(\mathfrak{D}(f, \sigma))>0} \operatorname{vol}\left(D_{i, a} \cap \mathfrak{D}(f, \sigma)\right) .
$$

From now on, the aim is to show that (5) is a linear form in $a$. Under the assumption that the conditions $\operatorname{Pr}(f, \sigma)>0$ and $\operatorname{vol}(\mathfrak{D}(f, \sigma))>0$ are equivalent, it means that (6) vanishes and the distribution of $r_{i} / p$ is uniform.

We assume still that a polynomial $f(x)$ has no rational roots, and put

$$
\begin{aligned}
& \mathbb{Q}(f):=\mathbb{Q}\left(\alpha_{1}, \ldots, \alpha_{n}\right), \\
& \beta_{i}:=\alpha_{i}-\operatorname{tr}_{\mathbb{Q}(f) / \mathbb{Q}}\left(\alpha_{i}\right) /[\mathbb{Q}(f): \mathbb{Q}] \quad(\neq 0), \\
& L R_{0}:=\left\{\left(l_{1}, \ldots, l_{n}\right) \in \mathbb{Q}^{n} \mid \sum_{i=1}^{n} l_{i} \alpha_{i} \in \mathbb{Q}\right\} \\
& =\left\{\left(l_{1}, \ldots, l_{n}\right) \in \mathbb{Q}^{n} \mid \sum_{i=1}^{n} l_{i} \beta_{i}=0\right\}, \\
& \mathfrak{D}_{f}:=\left\{\boldsymbol{x} \in \mathbb{R}^{n} \mid(\boldsymbol{l}, \boldsymbol{x}) \in \mathbb{Z} \quad \text { for }{ }^{\forall} \boldsymbol{l} \in L R_{0} \cap \mathbb{Z}^{n}\right\} .
\end{aligned}
$$




\section{YOSHIYUKI KITAOKA}

Lemma 0.1. Suppose that $\alpha_{i}-\alpha_{j} \notin \mathbb{Q}$ for any distinct $i, j$. We have

$$
\sum_{i=1}^{n} \sum_{\sigma \in S_{n}} \operatorname{vol}\left(D_{i, a} \cap \mathfrak{D}(f, \sigma)\right)=\sum_{i=1}^{n} \operatorname{vol}\left(\left\{\boldsymbol{x} \in[0,1)^{n} \mid x_{i} \leq a\right\} \cap \mathfrak{D}_{f}\right),
$$

in particular,

$$
\sum_{\sigma \in S_{n}} \operatorname{vol}(\mathfrak{D}(f, \sigma))=\operatorname{vol}\left([0,1)^{n} \cap \mathfrak{D}_{f}\right)
$$

Pro of. The equations

$$
\begin{aligned}
& D_{i, a} \cap \mathfrak{D}(f, \sigma) \\
& =\left\{\boldsymbol{x} \in[0,1)^{n} \mid x_{1} \leq \cdots \leq x_{n}, x_{i} \leq a,\left(\boldsymbol{l}, \sigma^{-1}(\boldsymbol{x})\right) \in \mathbb{Z} \quad \text { for } \quad{ }^{\forall} \in L R_{0} \cap \mathbb{Z}^{n}\right\} \\
& =\left\{\boldsymbol{x} \in[0,1)^{n} \mid x_{1} \leq \cdots \leq x_{n}, x_{i} \leq a, \sigma^{-1}(\boldsymbol{x}) \in \mathfrak{D}_{f}\right\} \\
& =\sigma\left(\left\{\boldsymbol{y} \in[0,1)^{n} \mid y_{\sigma^{-1}(1)} \leq \cdots \leq y_{\sigma^{-1}(n)}, y_{\sigma^{-1}(i)} \leq a\right\} \cap \mathfrak{D}_{f}\right)
\end{aligned}
$$

imply

$$
\begin{aligned}
& \operatorname{vol}\left(D_{i, a} \cap \mathfrak{D}(f, \sigma)\right) \\
& =\operatorname{vol}\left(\left\{\boldsymbol{y} \in \mathbb{R}^{n} \mid 0<y_{\sigma^{-1}(1)}<\cdots<y_{\sigma^{-1}(n)}<1, y_{\sigma^{-1}(i)} \leq a\right\} \cap \mathfrak{D}_{f}\right) .
\end{aligned}
$$

Here, we note that by the assumption, $L R_{0}$ contains no vector of the form $(0, \ldots, 0,1,0, \ldots,-1,0, \ldots, 0)$ and $\mathfrak{D}_{f}$ is defined by $t$ linearly independent vectors $\boldsymbol{m}_{j}$ and the volume vol is $(n-t)$-dimensional one, hence it is not necessary to care an additional equation $x_{i}=x_{j}$ for $i \neq j$. Let us define a mapping $\phi$ from

$$
X(i):=\left\{(\boldsymbol{x}, i) \mid \boldsymbol{x} \in(0,1)^{n}, 1 \leq i \leq n, x_{i} \leq a, x_{j} \neq x_{l} \quad \text { if } j \neq l\right\}
$$

to the union of

$$
Y(\sigma, k):=\left\{(\boldsymbol{y}, \sigma, k) \mid \boldsymbol{y} \in(0,1)^{n}, 0<y_{\sigma(1)}<\cdots<y_{\sigma(n)}<1, y_{\sigma(k)} \leq a\right\}
$$

by $\phi((\boldsymbol{x}, i))=(\boldsymbol{x}, \sigma, k)$, where $\sigma, k$ are defined by

hence

$$
x_{\sigma(1)}<\cdots<x_{\sigma(n)}, k=\sigma^{-1}(i),
$$

$$
\phi(X(i))=\cup_{\sigma, k: \sigma(k)=i} Y(\sigma, k) \text { for } i=1, \ldots, n .
$$

The mapping is clearly bijective for any fixed $i$, and if $(\boldsymbol{y}, \sigma, k) \in Y(\sigma, k)$ and $\left(\boldsymbol{y}, \sigma^{\prime}, k^{\prime}\right) \in Y\left(\sigma^{\prime}, k^{\prime}\right)$ occur with $\sigma(k)=\sigma^{\prime}\left(k^{\prime}\right)$, then $\sigma=\sigma^{\prime}, k=k^{\prime}$ hold. Hence, defining $\operatorname{pr}$ by $\operatorname{pr}(\boldsymbol{y}, \sigma, k)=\boldsymbol{y}$, we have

$$
\operatorname{pr}(Y(\sigma, k)) \cap \operatorname{pr}\left(Y\left(\sigma^{\prime}, k^{\prime}\right)\right)=\emptyset
$$

if $\sigma(k)=\sigma^{\prime}\left(k^{\prime}\right)$ and either $\sigma \neq \sigma^{\prime}$ or $k \neq k^{\prime}$, and so 


$$
\begin{aligned}
& \sum_{i=1}^{n} \sum_{\sigma} \operatorname{vol}\left(D_{i, a} \cap \mathfrak{D}(f, \sigma)\right) \\
& =\sum_{i=1}^{n} \sum_{\sigma} \operatorname{vol}\left(\operatorname{pr}\left(Y\left(\sigma^{-1}, i\right)\right) \cap \mathfrak{D}_{f}\right) \\
& =\sum_{k=1}^{n} \sum_{\sigma} \operatorname{vol}\left(\operatorname{pr}(Y(\sigma, k)) \cap \mathfrak{D}_{f}\right) \\
& =\sum_{i} \sum_{k, \sigma: \sigma(k)=i} \operatorname{vol}\left(\operatorname{pr}(Y(\sigma, k)) \cap \mathfrak{D}_{f}\right) \\
& =\sum_{i} \operatorname{vol}\left(\cup_{k, \sigma: \sigma(k)=i} p r(Y(\sigma, k)) \cap \mathfrak{D}_{f}\right) \\
& =\sum_{i} \operatorname{vol}\left(p r\left(\cup_{k, \sigma: \sigma(k)=i} Y(\sigma, k)\right) \cap \mathfrak{D}_{f}\right) \\
& =\sum_{i} \operatorname{vol}\left(p r(X(i)) \cap \mathfrak{D}_{f}\right) \\
& =\sum_{i} \operatorname{vol}\left(\left\{\boldsymbol{x} \in[0,1)^{n} \mid x_{i} \leq a\right\} \cap \mathfrak{D}_{f}\right)
\end{aligned}
$$

Hence to show the equidistribution, we have only to see that each factor $\operatorname{vol}\left(\left\{\boldsymbol{x} \in[0,1)^{n} \mid x_{i} \leq a\right\} \cap \mathfrak{D}_{f}\right)$ is a linear form in $a$.

Lemma 0.2. Suppose that $\beta_{1}, \ldots, \beta_{r}$ are linearly independent over $\mathbb{Q}$ and

$$
\left(\beta_{r+1}, \ldots, \beta_{n}\right)=\left(\beta_{1}, \ldots, \beta_{r}\right) T \quad \text { for }{ }^{\exists} T \in M_{r, n-r}(\mathbb{Q}) .
$$

Then we have $r=n-t$,

$$
L R_{0}=\left\{\left(l_{1}, \ldots, l_{n}\right) \in \mathbb{Q}^{n} \mid\left(\begin{array}{c}
l_{1} \\
\vdots \\
l_{r}
\end{array}\right)=-T\left(\begin{array}{c}
l_{r+1} \\
\vdots \\
l_{n}
\end{array}\right)\right\}
$$

and

$$
\left(\begin{array}{c}
\boldsymbol{m}_{1} \\
\vdots \\
\boldsymbol{m}_{t}
\end{array}\right)=\left(S^{t} T,-S\right) \quad \text { for }{ }^{\exists} S \in G L_{n-r}(\mathbb{Q}) \cap M_{n-r}(\mathbb{Z})
$$


Proof. By

$$
\sum_{i=1}^{n} x_{i} \beta_{i}=\left(\beta_{1}, \ldots, \beta_{r}\right)\left\{\left(\begin{array}{c}
x_{1} \\
\vdots \\
x_{r}
\end{array}\right)+T\left(\begin{array}{c}
x_{r+1} \\
\vdots \\
x_{n}
\end{array}\right)\right\} \quad\left(x_{i} \in \mathbb{R}\right),
$$

we see

$$
\begin{aligned}
L R_{0} & =\left\{\left(l_{1}, \ldots, l_{n}\right) \in \mathbb{Q}^{n} \mid \sum_{i=1}^{n} l_{i} \beta_{i}=0\right\} \\
& =\left\{\left(l_{1}, \ldots, l_{n}\right) \in \mathbb{Q}^{n} \mid\left(\begin{array}{c}
l_{1} \\
\vdots \\
l_{r}
\end{array}\right)=-T\left(\begin{array}{c}
l_{r+1} \\
\vdots \\
l_{n}
\end{array}\right)\right\},
\end{aligned}
$$

which implies $t=\operatorname{dim} L R_{0}=n-r$. On the other hand, the definition of $T$ implies

$$
\left({ }^{t} T,-1_{n-r}\right)\left(\begin{array}{c}
\beta_{1} \\
\vdots \\
\beta_{n}
\end{array}\right)=0^{(n-r, 1)},
$$

hence row vectors of the matrix $\left({ }^{t} T,-1_{n-r}\right)$ spann $L R_{0}$ by $\operatorname{rank}\left({ }^{t} T,-1_{n-r}\right)=$ $\operatorname{dim} L R_{0}$ and so there is a matrix $S \in G L_{n-r}(\mathbb{Q})$ such that

$$
\left(\begin{array}{c}
\boldsymbol{m}_{1} \\
\vdots \\
\boldsymbol{m}_{t}
\end{array}\right)=S\left({ }^{t} T,-1_{n-r}\right)=\left(S^{t} T,-S\right)
$$

which implies that $S$ is integral.

Lemma 0.3. Supposing the assumption on $\beta_{i}$ in Lemma 0.2, we have

$$
\begin{aligned}
\mathfrak{D}_{f} & =\left\{\boldsymbol{x} \in \mathbb{R}^{n} \mid\left(S^{t} T,-S\right)^{t} \boldsymbol{x} \in M_{t, 1}(\mathbb{Z})\right\} \\
& =\left\{\boldsymbol{x} \in \mathbb{R}^{n} \mid\left(x_{r+1}, \ldots, x_{n}\right)=\left(x_{1}, \ldots, x_{r}\right) T+\boldsymbol{k}^{t} S^{-1}\left({ }^{\exists} \boldsymbol{k} \in M_{1, t}(\mathbb{Z})\right)\right\} .
\end{aligned}
$$

For $\left(x_{1}, \ldots, x_{r}\right) \in[0,1)^{r}$, there are exactly $|\operatorname{det}(S)|$ vectors $\left(x_{r+1}, \ldots, x_{n}\right) \in$ $[0,1)^{n-r}$ such that $\left(x_{1}, \ldots, x_{n}\right) \in \mathfrak{D}_{f}$. 
Proof. We see that

$$
\begin{aligned}
\mathfrak{D}_{f} & =\left\{\boldsymbol{x} \in \mathbb{R}^{n} \mid\left(\begin{array}{c}
\boldsymbol{m}_{1} \\
\vdots \\
\boldsymbol{m}_{t}
\end{array}\right){ }^{t} \boldsymbol{x} \in M_{t, 1}(\mathbb{Z})\right\}=\left\{\boldsymbol{x} \in \mathbb{R}^{n} \mid\left(S^{t} T,-S\right)^{t} \boldsymbol{x} \in M_{t, 1}(\mathbb{Z})\right\} \\
& =\left\{\boldsymbol{x} \in \mathbb{R}^{n} \mid\left(\left(x_{r+1}, \ldots, x_{n}\right)-\left(x_{1}, \ldots, x_{r}\right) T\right)^{t} S \in M_{1, t}(\mathbb{Z})\right\} \\
& =\left\{\boldsymbol{x} \in \mathbb{R}^{n} \mid\left(x_{r+1}, \ldots, x_{n}\right)=\left(x_{1}, \ldots, x_{r}\right) T+\boldsymbol{k}^{t} S^{-1}\left({ }^{\exists} \boldsymbol{k} \in M_{1, t}(\mathbb{Z})\right)\right\} .
\end{aligned}
$$

Since $S \in G L_{n-r}(\mathbb{Q})$ is an integral regular matrix, the inclusion $\mathbb{Z}^{n-r}{ }^{t} S^{-1} \supset$ $\mathbb{Z}^{n-r}$ is clear. Hence, for $\left(x_{1}, \ldots, x_{r}\right) \in \mathbb{R}^{r}$, there is a vector $\left(x_{r+1}, \ldots, x_{n}\right):=$ $\left(x_{1}, \ldots, x_{r}\right) T+\boldsymbol{k}^{t} S^{-1} \in[0,1)^{n-r}$ for some integral vector $\boldsymbol{k}$. The number of such integral vectors is $|\operatorname{det}(S)|$ since $S$ is integral.

Thus we see that, assuming that $\beta_{1}, \ldots, \beta_{r}$ is linearly independent over $\mathbb{Q}$ and $1 \leq i \leq r$ as above

$$
\begin{aligned}
& \left\{\boldsymbol{x} \in[0,1)^{n} \mid x_{i} \leq a\right\} \cap \mathfrak{D}_{f}= \\
& \left\{\boldsymbol{x} \in[0,1)^{n} \mid x_{i} \leq a,\left(x_{r+1}, \ldots, x_{n}\right)=\left(x_{1}, \ldots, x_{r}\right) T+\boldsymbol{k}^{t} S^{-1}\left({ }^{\exists} \boldsymbol{k} \in M_{1, t}(\mathbb{Z})\right)\right\},
\end{aligned}
$$

which is included in the union of sets parallel to the subspace

$$
\left\{\boldsymbol{x} \in \mathbb{R}^{n} \mid\left(x_{r+1}, \ldots, x_{n}\right)=\left(x_{1}, \ldots, x_{r}\right) T\right\},
$$

and the projection to $E_{i, a}:=\left\{\boldsymbol{x} \in[0,1)^{r} \mid x_{i} \leq a\right\}$ is exactly $|\operatorname{det}(S)|$-fold independently on $\boldsymbol{x}$ by the lemma above. Hence the volume of $\left\{\boldsymbol{x} \in[0,1)^{n} \mid\right.$ $\left.x_{i} \leq a\right\} \cap \mathfrak{D}_{f}$ as an $r(=n-t)$-dimensional set is proportional to $a=\operatorname{vol}\left(E_{i, a}\right)$. In general, for given $i$, we have only to take a subset $j_{1}, \ldots, j_{r}$ such that $\beta_{j_{1}}, \ldots, \beta_{j_{r}}$ are linearly independent over $\mathbb{Q}$ and $j_{k}=i$ for some $k$. Thus, we have proved that

Theorem 0.1. If a polynomial $f(x)$ has no rational root, $\alpha_{i}-\alpha_{j} \notin \mathbb{Q}$ for any distinct $i, j$, and the conditions $\operatorname{Pr}(f, \sigma)>0$ and $\operatorname{vol}(\mathfrak{D}(f, \sigma))>0$ are equivalent, then Conjectures 1, 2 imply the equidistribution of $r_{i} / p$ for local roots $r_{i}$ of the polynomial.

So, the equidistribution of $r_{i} / p$ for local roots $r_{i}$ is likely for an irreducible polynomial $f(x)$ of $\operatorname{deg} f>1$. Although the polynomial

$$
f(x)=\left(x^{2}-2\right)\left((x-1)^{2}-2\right)
$$

does not satisfy the assumption of the theorem, the equidistribution of local roots $r_{i} / p$ is true by [DFI], [T]. 


\section{YOSHIYUKI KITAOKA}

Put, for an algebraic number field $F$ containing $\mathbb{Q}(f)$

$$
\operatorname{Spl}(f, F):=\{p \in \operatorname{Spl}(f) \mid p \text { is fully splitting at } F\} \text {. }
$$

If a subsequence of $r_{i} / p$ for local roots $r_{i}$ of $f$ restricted on $p \in \operatorname{Spl}(f, F)$ distributes uniformly for every irreducible polynomial $f$ of $\operatorname{deg} f>1$ and every number field $F$, then the one-dimensional distribution of $r_{i} / p$ for any reducible polynomial without rational root is true.

REMARK. Foo proves in $[\mathrm{F}]$ that the set $\left\{r_{i} / p \mid 1 \leq i \leq n, p \in \operatorname{Spl}(f)\right\}$ is dense in the interval $(0,1)$ for an irreducible polynomial $f(x)$ of degree $>1$ under the Bouniakowsky conjecture.

\section{REFERENCES}

[DFI] DUKE, W.-FRIEDLANDER, J. B.-IWANIEC, H.: Equidistribution of roots of a quadratic congruence to prime moduli, Ann. Math. 141 (1995), 423-441.

[F] FOO, T.: The Bouniakowsky conjecture and the density of polynomial roots to prime moduli, Acta Arith. 144 (2010), 1-4.

[K1] KITAOKA, Y.: Notes on the distribution of roots modulo a prime of a polynomial, Unif. Distrib. Theory 12 (2017), no. 2, 91-116.

[K2] KITAOKA, Y.: Statistical distribution of roots of a polynomial modulo primes III, International Journal of Statistics and Probability 7 (2017), 115-124.

[K3] KITAOKA, Y.: Notes on the distribution of roots modulo a prime of a polynomial II, Unif. Distrib. Theory 14 (2019), no. 1, 87-104.

[K4] KITAOKA, Y.: Conjectures on the distribution of roots modulo a prime of a polynomial, arXiv : $1905.02364 \mathrm{v} 7$

[T] TÓTH, Á.: Roots of quadratic congruences, Internat. Math. Res. Notices, (2000), 719-739.

Received February 14, 2020

Accepted April 21, 2020

\section{Yoshiyuki Kitaoka}

(Author is retired)

Asahi

JAPAN

E-mail: kitaoka@meijo-u.ac.jp 